\title{
ESTIMATING VALUE-AT-RISK BASED ON NON-NORMAL DISTRIBUTIONS
}

Mária Bohdalová ${ }^{1}$, Michal Greguš ${ }^{2}$

\begin{abstract}
The article presents a comparative study of parametric linear value-at-risk (VaR) models used for estimating the risk of financial portfolios. We illustrate how to adjust VaR for auto-correlation in portfolio returns. The article presents static and dynamic methodology to compute VaR, based on the assumption that daily changes are independent and identically distributed (normal or non-normal) or auto-correlated in terms of the risk factor dynamics. We estimate the parametric linear VaR over a risk horizon of 1 day and 10 days at $99 \%$ and 95\% confidence levels for the same data. We compare the parametric VaR and a VaR obtained using Monte Carlo simulations with historical simulations and use the maximum likelihood method to calibrate the distribution parameters of our risk factors. The study investigated whether the parametric linear VaR applies to contemporary risk factor analysis and pertained to selected foreign rates.
\end{abstract}

JEL Classification Numbers: G10, G17, C13, C15, DOI: http://dx.doi.org/10.12955/cbup.v3.601

Keywords: Value at Risk, leptokurtic distribution, skewed distribution, normal mixture distribution, Monte Carlo simulation

\section{Introduction}

Instability now characterizes the contemporary financial market because of the global economic crisis. Correspondingly, academic literature offers a rich source of methods for analyzing the risk of financial products, based on mathematical models. The main aim of this article is to review the method for estimating VaR, based on non-normal distribution of the returns.

\section{Literature review}

During the 1990s, a new technology to measure and control financial risk, based on statistical techniques, emerged as the Value at Risk (VaR) methodology (Allen, 2003). This method applies primarily to two major risk types: market and credit. Market risk is the potential for change in market prices of an institution's holdings, which may adversely affect its financial security. Credit risk is the risk of economic loss from an obligor's failure to comply with terms and conditions of a contract or agreement, with failure due to financial inability or an unwillingness to perform, or both (Allen, 2003). The VaR concept is applicable to other types of risk, such as operational. Duffie and Pan (1997) and Jorion (2006) provide a general exposition of VaR. Financial institutions might use VaR to assess risks, while regulatory committees may use it to set marginal requirements. In either case, VaR is used to ensure financial institutions remain in business after catastrophic events. From the viewpoint of a financial institution, $\mathrm{VaR}$ is defined as the maximal loss of a financial position during a given time period $(h)$ for a given probability $(p)$. VaR is a measure of loss associated with a rare (or extraordinary) event under normal market conditions. Alternatively, from the viewpoint of a regulatory committee, $\mathrm{VaR}$ is the minimal loss under extraordinary market circumstances (Tsay, 2010). In real applications, the risk management probability $(p)$ is equal to 0.01 and, with stress testing, 0.001. A regulatory committee might set the time horizon $(h)$ as 1 day or 10 days for market risk, and 1 year or 5 years for credit risk. The daily frequency is often used for market risk analysis (Tsay, 2010).

For instance, assume that at a certain time index $(t)$, we are interested in the risk of a financial position for the next $(h)$ period. Let $\Delta V(h)$ be the change in value of the underlying assets of the financial position from time $t$ to $t+\Delta$ and let $L(h)$ the associated loss function. Measurements are in euros and

\footnotetext{
1 Mária Bohdalová, Faculty of Management, Comenius University in Bratislava, Slovakia, Maria.Bohdalova@fm.uniba.sk
2 Michal Greguš, Faculty of Management, Comenius University in Bratislava, Slovakia, Michal.Gregus@fm.uniba.sk
} 
they are random variables at time index, t. $L(h)$ is either a positive or a negative function of $\Delta V(h)$, depending on a short or long financial position. We denote the cumulative distribution function (CDF) of $\mathrm{L}(h)$ as $F_{h}(x)$. The VaR of a financial position over the time horizon $(h)$ with tail probability $(p)$ is defined as

$$
p=\operatorname{Pr}[L(h) \geq V a R]=1-\operatorname{Pr}[L(h)<\operatorname{VaR}]
$$

If the $\operatorname{CDF} F_{h}(x)$ of equation (1) is known then $1-p=\operatorname{Pr}[L(h)<V a R]$. According to Tsay (2010) and Bohdalová \& Greguš (2013), VaR is simply the $(1-p)$-th quantile of the CDF of the loss function $L(h)$. We note that $\operatorname{CDF} F_{h}(x)$ is the focus of the econometric modelling. Different methods for estimating the CDF give rise to different approaches for calculating VaR. As mentioned above, VaR is based on the upper tail of a loss function. Loss occurs when the returns are negative (investor takes a long financial position). Logarithmic returns correspond approximately to percentage changes in the value of a financial asset, and therefore, we use logarithmic returns $\left(r_{t}\right)$ in the data analysis. The VaR from the upper quantile of the distribution of $r_{t+1}$, based on information available at a time $(t)$, is therefore a percentage. The euro amount of VaR is the cash value of the financial position multiplied by the VaR of the logarithmic return series. That is,

$$
\mathrm{VaR} \text { amount }=\text { Invested amount } \times \mathrm{VaR} \text { percentage (of logarithmic returns). }
$$

VaR should be computed using the predictive distribution of the future return $\left(r_{t+1}\right)$ for the financial position, based on information available at a time $(t)$. Predictive distribution, taking into account the parameter uncertainty in a properly specified model, is difficult to obtain (Tsay, 2010). An alternative approach to calculating VaR uses the time-series econometric models.

Many implementations of VaR assume normally distributed asset returns. In today's economic conditions, asset returns have a heavy tailed distribution. In this article, we use alternative distributions that can better reflect the empirical evidence. The alternating depression and prosperity phases result in the normal mixture distribution being a better option for describing the analyzed asset returns.

\section{Parametric linear VaR}

The method for computing linear parametric VaR is derived from Jorion (2003, 2006), Alexander (2008), and Dowd (2002). The following is the formula for (1-p)-th $h$-day parametric VaR with uncorrelated or auto-correlated return of the first order:

$$
\operatorname{VaR}_{h, p}=\sqrt{\tilde{h}} F^{-1}(1-p) \sigma-h \mu,
$$

where $\mu$ and $\sigma$ are daily mean and standard deviation, respectively, $h$ is the horizon and $\tilde{h}$ is the horizon adapted for assuming auto-correlation $\rho$ of the first order.

$$
\tilde{h}=h+2 \frac{\rho}{(1-\rho)^{2}}\left[(h-1)(1-\rho)-\rho\left(1-\rho^{h-1}\right)\right],
$$

Equality $(\tilde{h}=h)$ is fulfilled when returns are not correlated.

A normal parametric VaR ensues when $F^{-1}(1-p)$ in equation (3) is the standard normal $(1-p)$ quantile value. Student's $t$ VaR are obtained from equation (3) for

$$
F^{-1}(1-p)=\sqrt{v^{-1}(v-2)} t_{v}^{-1}(1-p),
$$

where $t_{v}^{-1}(1-p)$ is $(1-p)$ quantile of the standard Student's $t$ distribution, with $v$ degrees of freedom $(v>2)$ (Dowd, 2002). Student's $t$ probability distribution function is used where data distribution is symmetric, unimodal, and without outliers. The density of the Student's $t$ distribution is leptokurtic, 
the peak in the center of the distribution is higher than the peak of the equivalent normal density, and the tails are heavier (Alexander, 2008).

Mixture distributions may better describe differing market regimes. For instance, when we use a mixture of the two normal distributions, we assume that there are two regimes for returns, i.e. mean $\mu_{1}$ and variance $\sigma_{1}^{2}$, and mean $\mu_{2}$ and variance $\sigma_{2}^{2}$. The last parameter of the mixture is probability, which is $\pi$ for the first regime and $1-\pi$ for the second. The distribution function of a mixture distribution is a probability-weighted sum of the component distribution functions (Alexander, 2008).

There is no explicit formula for computing $(1-p)$ quantile $F^{-1}(1-p)$ of mixture distributions. In this article, we assume that a mixture distribution involves two normal distributions or two Student's $t$ distributions. The value of the $(1-p)$ quantile is computed using an iterative approximation method (Alexander, 2008) or the Quantile [Distribution [parameters], 1-p] in Wolfram Mathematica software.

\section{Data and methodology}

This article analyses the risk of investing in two popular currencies, both in USD denomination. One is the digital currency, bitcoin, and the second is the euro. The euro is the single currency shared by 19 European Union's Member States, which together make up the euro area. The introduction of the euro in 1999 was a major step in European integration (European Commission, n.d.). Similar to many financial markets, the bitcoin is a decentralized virtual currency with no institution, such as a central bank, controlling its value (Easwaran, Dixit, \& Sinha, 2015). Bitcoin involves an online payment system introduced in 2009 for worldwide users.

We analyzed the risk of the closing spot price ( $P_{t}$ measured in time $\left.t\right)$ and its fluctuation (measured in terms of logarithmic return) for exchange rates of the bitcoin (BTC/USD) and the euro (EUR/USD). The analysis period was August 15, 2011 to March 31, 2015, which comprised 1,134 trading days, or $T=1,133$ logarithmic returns (log returns are obtained by formula: $r_{t}=\ln P_{t} / \ln P_{t-1}, t=1, \ldots, T$, excluding account dividends). A global financial portal provided the data (Fusion Media Ltd, n. d.). Our sample period is particularly relevant with several major changes occurring during that time.

The time series of the closing price and the effects of the currency crises are shown for the bitcoin (Figure 1) and for the euro (Figure 2). The bitcoin was at a historical low in July 2012, and the last decrease shown was in January 17, 2015. The largest deviation was at the end of the first quarter of 2013 (see logarithmic returns; Figure 1).

\begin{tabular}{l} 
Figure 1: Bitcoin (BTC/USD) closing price and logarithmic returns, \\
analysis period: August, 16, 2011 - March 31, 2015, daily data \\
\hline Source: Authors
\end{tabular}




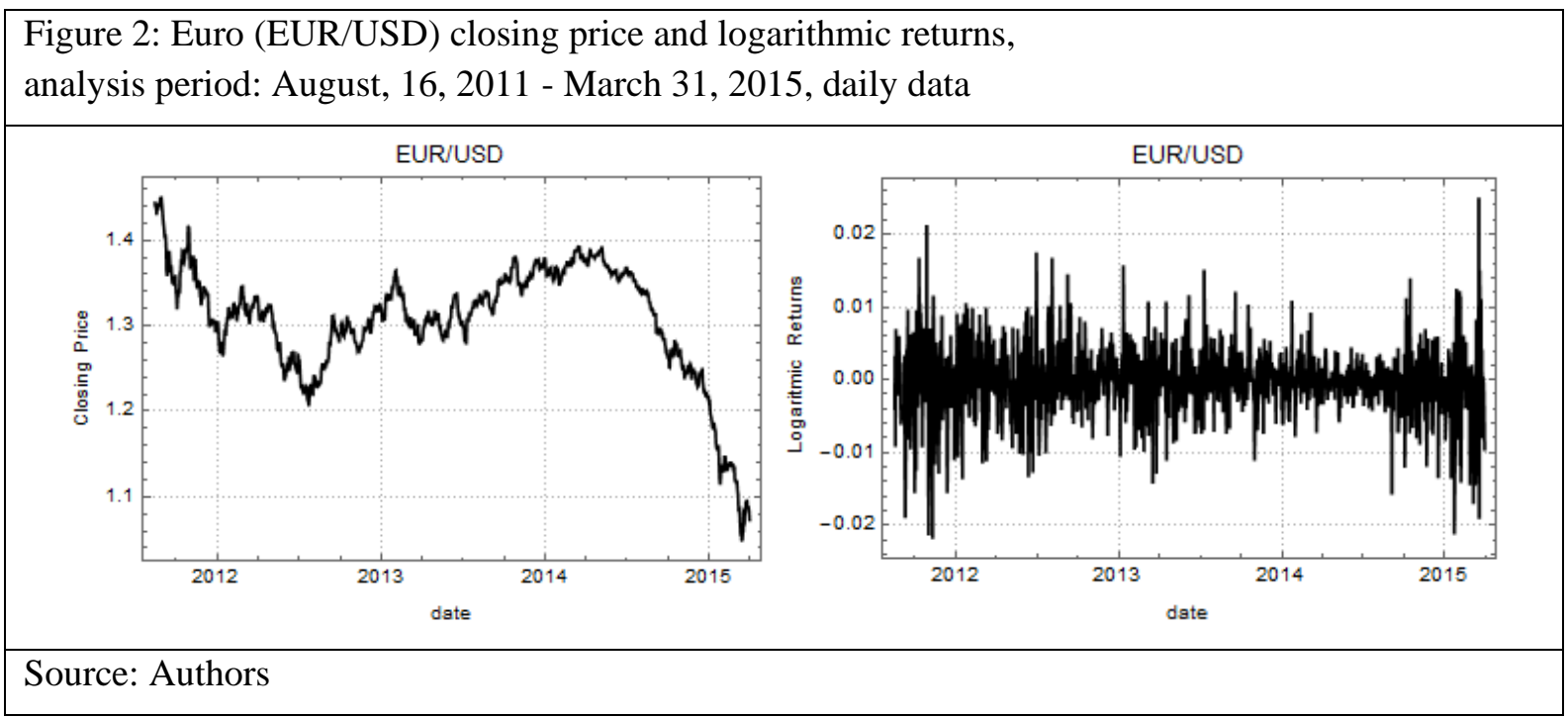

Growth of the euro foreign exchange rates, compared to those of the bitcoin, was more stable in the considered period. However, from January 2015 the euro reached a historic low (Figure 2). As published online, there are three reasons why the euro is sliding to a nine-year low (Cable News Network, 2015, 3 reasons the euro is plunging). Firstly, during the second half of 2014, the US dollar made significant gains against all other major global currencies, including the British pound, Swiss franc, and Japanese yen. This was due to a 5\% growth in the US economy in the third quarter of 2014, and subsequent job creation in the US. However, in the Eurozone, unemployment is still near record high, the economy has stagnated, and the risk of deflation looms largely. Secondly, the European Central Bank has implemented their quantitative easing policy to stimulate the economy and forestall the risk of deflation. Lastly, the result of the Greek elections in January 2015 could reinstate the Eurozone debt crisis.

Table 1 shows estimations of the descriptive statistics (column Estimate) for 1,133 data points, including approximate standard errors and the test statistics (ratio of the estimate of the statistics to its standard error), for the logarithmic returns of both foreign exchange rates. Test statistics is used to verify hypothesis $\mathrm{H}_{0}$ : statistics (mean, standard deviation, etc.) is equal to zero against hypothesis $\mathrm{H}_{\mathrm{A}}$ : statistics is unequal to zero (or statistics is significant). Over the sample period, the mean of the bitcoin logarithmic returns was positive, but with a significance level of 0.05 was equal to zero (test statistics is less than $1.96^{3}$, see Table 1 ). Also, first order auto-correlation was absent (test statistics is equal to $-4.9531 \mathrm{E}-1$, is less than 1.96 an therefore we can not to reject $\mathrm{H}_{0}$ ). Standard deviation appeared to be highly significant, skewness was significant and negative, and kurtosis was significant and positive. The euro logarithmic returns had a negative mean and negative first order auto-correlation, which were both significant for significance level $0.1^{4}$ (see Table 1). Skewness is equal to zero on the significance level 0.05. Standard deviation and positive excess kurtosis appears to be highly significant (test statistics are greater as 1.96), see Table 1.

Table 2 provides Cramér-von Mises $p$-values of the distribution fit test. This test rejects normal distribution of logarithmic returns for both currencies. A graphical comparison of the probability distributions (normal, Student's $t$, and normal mixture) and a histogram of the bitcoin and euro logarithmic returns are shown in Figures 3 and 4, which show the optimal distribution is a Student's $t$ distribution for bitcoin and normal mixture distribution for euro logarithmic returns.

\footnotetext{
${ }^{3} 97.5 \%$ quantile of the standardized normal distribution is equal to 1.96

${ }^{4} 95 \%$ quantile of the standardized normal distribution is equal to 1.65
} 
CBU I NTERNATIONAL CONFERENCE ON I NNOVATION, TECHNOLOGY TRANSFER AND EDUCATION MarCh 25-27, 2015, Prague, CZECH Republic

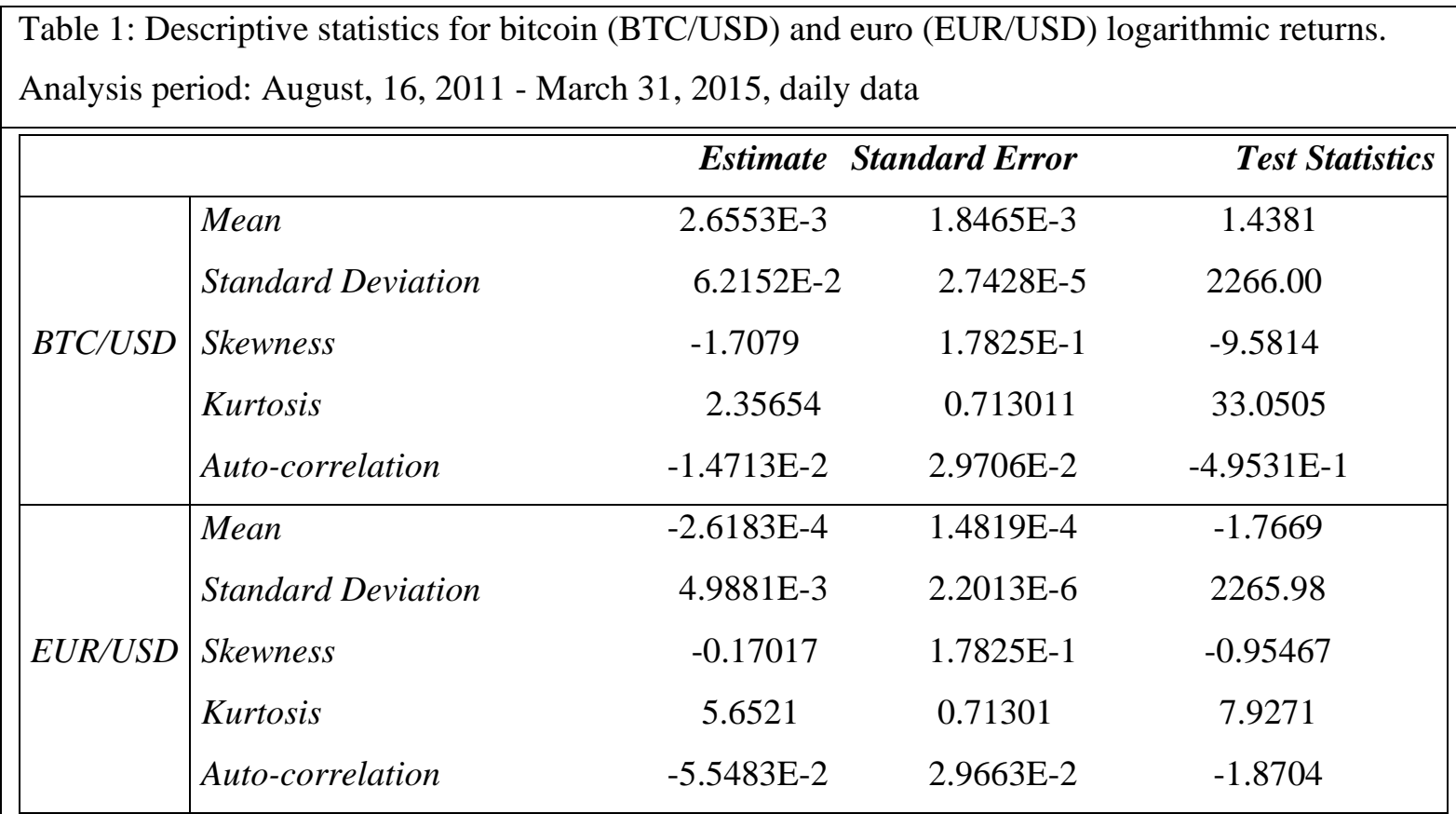

Source: Authors

Table 2: Distribution fit test for bitcoin (BTC/USD) and euro (EUR/USD) logarithmic returns (Cramér-von Mises p-values). Analysis period: August, 16, 2011 - March 31, 2015, daily data

\begin{tabular}{|l|r|r|}
\hline & BTC/USD & EUR/USD \\
\hline Student's t-Distribution & 0.2229 & 0.1786 \\
\hline Normal Mixture Distribution & 0.1003 & 0.6177 \\
\hline Normal Distribution & 0.0000 & 0.0000 \\
\hline
\end{tabular}

Source: Authors

Figure 3: Graphical comparison of the selected distributions for bitcoin (BTC/USD) logarithmic returns. Analysis period: August, 16, 2011 - March 31, 2015, daily data

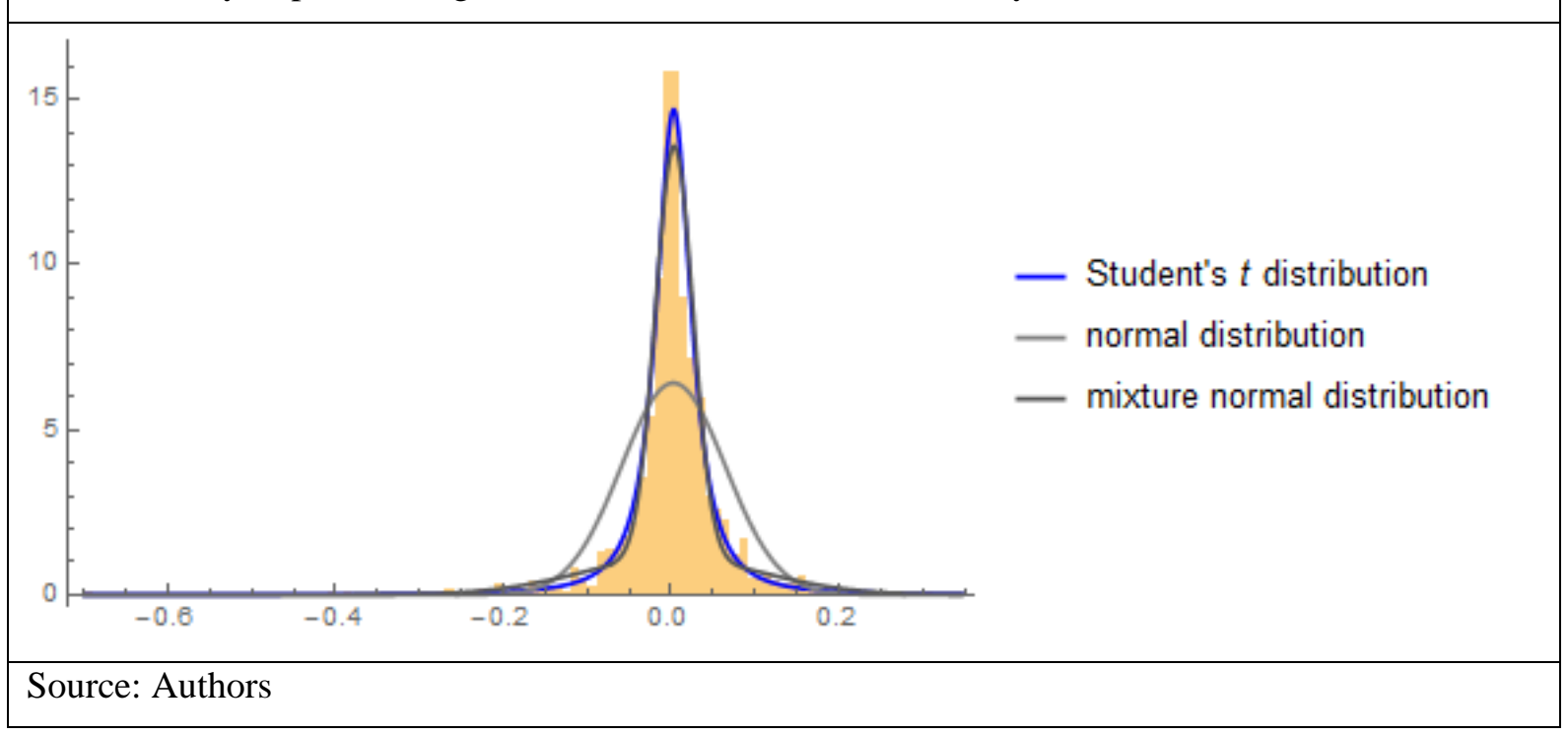


Figure 4: Graphical comparison of the selected distributions for euro (EUR/USD) logarithmic returns. Analysis period: August, 16, 2011 - March 31, 2015, daily data

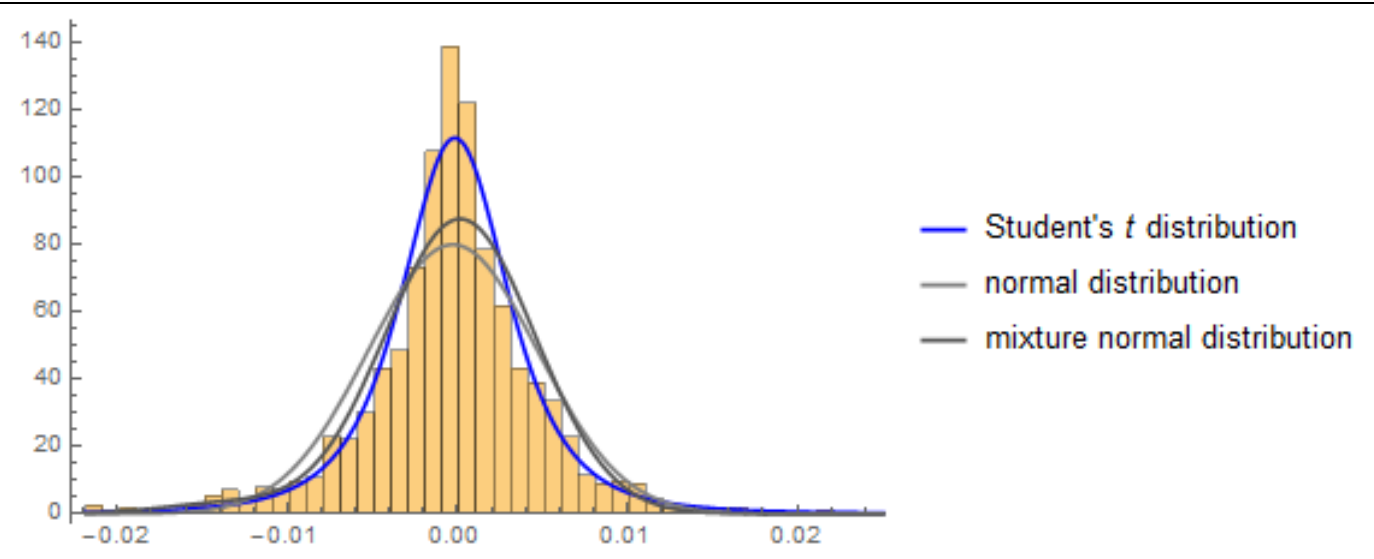

Source: Authors

Model parameters for all considered distributions were estimated using a maximum likelihood estimation (MLE) method and calibrated in Wolfram Mathematica software. The estimated parameters are given in Table 3. The normal mixture distribution assumed two normal components, one to represent the volatile regime and the second a stable trend. We found that for the bitcoin logarithmic returns, a normal mixture distribution assumed only $30 \%$ of the volatile regime. In the case of the euro logarithmic returns, the normal mixture distribution assumed $56 \%$ of the volatile regime, with smaller mean returns and approximately three times the volatility in comparison to the stable period.

Table 3: Parameters of the selected distributions for bitcoin (BTC/USD) and euro (EUR/USD) logarithmic returns. Analysis period: August, 16, 2011 - March 31, 2015, daily data

\begin{tabular}{|l|c|r|r|}
\hline \multirow{4}{*}{ Student's t distribution } & & BTC/USD & EUR/USD \\
\hline \multirow{5}{*}{ Normal Mixture Distribution } & $\mu$ & 0.0031 & -0.0001 \\
\cline { 2 - 4 } & $\sigma$ & 0.0234 & 0.0033 \\
\cline { 2 - 4 } & $v$ & 1.6135 & 3.0451 \\
& $\pi$ & 0.3002 & 0.5614 \\
\cline { 2 - 4 } & $\mu_{1}$ & 0.0004 & -0.0004 \\
\cline { 2 - 4 } & $\sigma_{1}$ & 0.1081 & 0.0064 \\
\cline { 2 - 4 } & $\mu_{2}$ & 0.0036 & -0.0001 \\
\cline { 2 - 4 } & $\sigma_{2}$ & 0.0223 & 0.0019 \\
\hline \multirow{3}{*}{ Normal Distribution } & $\mu$ & 0.0027 & -0.0003 \\
\hline Source: Authors & $\sigma$ & 0.0621 & 0.0050 \\
\hline
\end{tabular}

\section{Results and discussion}

Table 4 shows VaR for a simple linear exposure for the amounts of 1000 BTC and 1000 EUR. The normal, Student's $t$, and normal mixture distributions provide the estimates of the parametric VaR and the VaR obtained using Monte Carlo simulation. We modeled risk from these options of the risk factor distributions, estimating the $1 \%$ (5\%) 10-day VaR with the following assumptions about the currency 
dynamics: the daily logarithmic returns are independent and identically distributed or auto-correlated, or both. All estimates are based on the same data sample.

Table 4: 1\% (5\%) 10-day VaR estimations based on different distributions for invested amount 1000 bitcoin (BTC/USD) and 1000 euro (EUR/USD)

\begin{tabular}{|c|c|c|c|c|c|c|}
\hline \multirow{2}{*}{ Distribution } & & \multirow[b]{2}{*}{ Assumption } & \multicolumn{2}{|c|}{ Parametric VaR } & \multicolumn{2}{|c|}{ "Monte Carlo VaR } \\
\hline & & & BTC/USD & EUR/USD & BTC/USD & EUR/USD \\
\hline \multirow{4}{*}{ Student's $t$} & \multirow{2}{*}{ 1\% 10-day VaR } & iid $^{1}$ & 731.814 & 43.683 & 768.443 & 46.347 \\
\hline & & autocor. $^{2}$ & 722.185 & 42.998 & 666.988 & 43.963 \\
\hline & \multirow{2}{*}{ 5\% 10-day VaR } & iid & 265.596 & 24.912 & 256.134 & 24.814 \\
\hline & & autocor. & 262.101 & 24.522 & 238.598 & 24.900 \\
\hline \multirow{4}{*}{ Normal } & \multirow{2}{*}{ 1\% 10-day VaR } & iid & 457.201 & 36.698 & 445.934 & 36.345 \\
\hline & & autocor. & 451.185 & 34.908 & 440.066 & 35.774 \\
\hline & \multirow{2}{*}{ 5\% 10-day VaR } & iid & 323.258 & 25.948 & 307.307 & 26.015 \\
\hline & & autocor. & 319.005 & 24.683 & 303.263 & 25.606 \\
\hline \multirow{4}{*}{$\begin{array}{l}\text { Normal } \\
\text { Mixture }\end{array}$} & \multirow{2}{*}{ 1\% 10-day VaR } & iid & 631.092 & 36.191 & 625.149 & 36.628 \\
\hline & & autocor. & 622.842 & 35.615 & 597.496 & 37.075 \\
\hline & \multirow{2}{*}{ 5\% 10-day VaR } & iid & 334.993 & 25.444 & 337.351 & 26.547 \\
\hline & & autocor. & 330.64 & 25.037 & 334.715 & 26.437 \\
\hline
\end{tabular}

Source: Authors

${ }^{1}$ Assumption about independent and identically distributed logarithmic returns

${ }^{2}$ Assumption about presence of the autocorrelation of the first order in logarithmic returns

Historically, the 99\% and 95\% 10-day VaR for bitcoin was 633.357 and 263.486 BTC, respectively, and for the euro this was 42.302 and 26.907 EUR, respectively. Parametric VaR estimations gave comparable $\mathrm{VaR}$ values to that obtained using Monte Carlo simulations. VaR computed using normal distribution can give undervalued or overvalued results because of this distribution does not take into account heavy tails (assumption about normality of logarithmic return was rejected for both currency). Negative auto-correlations decreased the VaR. Our analyses show that investing in bitcoin is riskier than investing in the euro. For example, according to $99 \%$ of the 10-day VaR from the Student's $t$ distribution, we might lose about $73.182 \%$ of money invested in bitcoin and, although the euro recorded an increase in volatility, starting in January 2015, our loss by investing in the euro would be only $4.368 \%$.

\section{Conclusion}

Our results from the programming of Wolfram Mathematica ${ }^{5}$ showed that an investor who invests money in bitcoin must consider the high fluctuations in its exchange rate, which many factors affect. For example, the News cycle influences the rate of adoption, as do the geopolitical proceedings and statements that governments are likely to regulate bitcoin. The bitcoin may also fluctuate against 'fiat currencies' because of its perceived store of value compared to that of the fiat currency. Bitcoin has properties that make it similar to gold. Its volatility is driven largely by varying perceptions of the intrinsic value of cryptocurrency as a store of value and a method of value transfer. Bitcoin might be

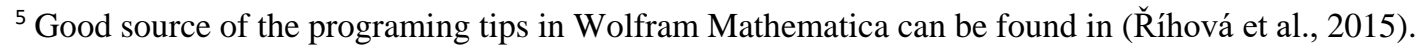


interesting as a currency in the countries that are currently experiencing financial crisis, but it will depend on its volatility in the economy of these countries versus its volatility in the US economy.

According to Barker (2015), bitcoin presents a variety of opportunities that were non-existent prior to its development. The recent acknowledgement by the internal revenue service (IRS) that bitcoin is an asset for tax purposes has clarified the situation for investors, and the promise of frictionless value transfer suggests innovative use in cases of foreign direct investment. In the near future, much of the volatility could be driven by investor perception of the ability of gateways to safeguard individual holdings, and provide for a reliable store of value as adoption increases.

\section{Acknowledgment}

This research was supported by a VUB grant, no. 2015-3-02/5 "Development of the theory and application of stochastic analysis of financial markets to the current problems of business practice (AMSAFT)."

\section{References}

Allen, S. (2003). Financial risk management. A practitioner's guide to managing market and credit risk. New Jersey: John Wiley \& Sons.

Alexander, C. (2008). Market risk analysis. Chichester: John Wiley \& Sons.

Barker, J.T. (2015). Why is bitcoin's value so volatile? Investopedia, LLC. Retrieved May 20, 2015 from http://www.investopedia.com/articles/investing/052014/why-bitcoins-value-so-volatile.asp

Bohdalová, M., \& Greguš, M. (2013). VaR based risk management. In P. Hájek (Ed.), CBU International Conference Proceedings (pp. 25-33). Prague, Czeck Republic Central Bohemia University. doi: 10.12955/cbup.2013.11

Cable News Network (2015, January 5). 3 reasons the euro is plunging [CNN Money update]. Retrieved July 26, 2015 from http://money.cnn.com/2015/01/05/investing/euro-slump-deepens/index.html

Dowd, K. (2002). An introduction to market risk measurement. Chichester: John Wiley \& Sons.

Duffie, D., \& Pan, J. (1997). An overview of value at risk. Journal of Derivatives, Spring, 7-48.

Easwaran, S., Dixit, M., \& Sinha, S. (2015). Bitcoin dynamics: the inverse square law of price fluctuations and other stylized facts. In F. Abergel, H. Aoyama, B. K. Chakrabarti, A. Chakraborti \& A. Ghosh (Eds.) Econophysics and data driven modelling of market dynamics (pp. 121-128). Switzerland: Springer International Publishing. doi: 10.1007/978-3-319-084732 _4

European Commission. (n.d.). Economic and financial affairs. Retrieved July 26, 2015 from http://ec.europa.eu/economy_ finance/euro/index_en.html

Fusion Media Ltd. (n.d.). Currency Rates. Retrieved from http://www.investing.com/currencies

Jorion, P. (2003). Financial risk manager handbook. New Jersey: John Wiley \& Sons.

Jorion, P. (2006). Value at risk: the new benchmark for managing financial risk. New York: McGraw-Hill.

Ř́ihová, V., Mošová, V., Valentová, I., Ř́na, J., Slezák, V., Pászto, V., Chmela, L., Zielina, M., \& Smrčka, D. (2015).

Software mathematica for economist. Olomouc: EPAVA.

Tsay, R. S. (2010). Analysis of financial times series. New Jersey: John Wiley \& Sons. 